\title{
Very Short-term Conflict Intensity Estimation Using Fisher Vectors
}

\author{
Gábor Gosztolya ${ }^{1,2}$ \\ ${ }^{1}$ MTA-SZTE Research Group on Artificial Intelligence, Szeged, Hungary \\ ${ }^{2}$ University of Szeged, Institute of Informatics, Szeged, Hungary \\ ggabor a inf.u-szeged.hu
}

\begin{abstract}
The automatic detection of conflict situations from human speech has several applications like obtaining feedback of employees in call centers, the surveillance of public spaces, and other roles in human-computer interactions. Although several methods have been developed to automatic conflict detection, they were designed to operate on relatively long utterances. In practice, however, it would be beneficial to process much shorter speech segments. With the traditional workflow of paralinguistic speech processing, this would require properly annotated training and testing material consisting of short clips. In this study we show that Support Vector Regression machine learning models using Fisher vectors as features, even when trained on longer utterances, allow us to efficiently and accurately detect conflict intensity from very short audio segments. Even without having reliable annotations of these such short chunks, the mean scores of the predictions corresponding to short segments of the same original, longer utterances correlate well to the reference manual annotation. We also verify the validity of this approach by comparing the SVM predictions of the chunks with a manual annotation for the full and the 5-second-long cases. Our findings allow the construction of conflict detection systems having smaller delay, therefore being more useful in practice.
\end{abstract}

Index Terms: conflict intensity estimation, computational paralinguistics, Fisher vectors

\section{Introduction}

Conflicts are an inherent part of everyday human communication, either in personal or in public life. In a conflicted situation, the people involved are pursuing incompatible goals [1,2]. This usually leads to conversations being more intense than usual, manifesting itself in raised voices and in a greater number of interruptions [3]. Since conflicts are one of the main causes of stress [4], the quick and automatic detection of conflicted situations could prove to be useful. With the rise of socially intelligent technologies, the automatic detection of conflicts could be the first step of handling them properly.

Of course, participating in a conflict affects the speech of people as well. For example, in such situations speakers tend to interrupt each other more frequently than usual, which leads to a more frequent occurrence of overlapping speech [5, 6], and also to increased volume and articulation tempo [7]. Over the past few years, researchers have applied various approaches for detecting conflict from the speech signal, ranging from the automatic detection of speech interruptions to Recurrent Deep Neural Networks $[3,7,8,9,10,11,12,13]$. What was common in these studies, however, is that (despite some authors using phrases such as 'continuous' and 'short-term') the actual' conflict intensity was determined based on a relatively long audio clip. The reason for this was simple: the absence of annotated short audio clips. For example, the most popular dataset used in these experiments is the SSPNet Conflict Corpus [3], which consists of 30-second-long segments. Although short(er) audio clips can easily be created automatically from longer utterances, without a proper annotation these would be of a very limited use in two distinct steps. The more straightforward issue is that we would need these recordings for model training. However, perhaps a more important issue with the absence of such short, annotated recordings is that of model evaluation: without appropriate development and test sets it is very hard to rate the performance of our classification or regression models.

In this study, in contrast with previous works, we will show that it is possible to train machine learning methods which can deliver a good performance even on very short utterances (in our case, even one second). For this, we employ the feature extraction approach of Fisher vectors (FV, [14]), which was shown to provide a robust utterance-level feature representation regardless of the length of the actual recording. Besides image processing studies, FVs were used in audio processing as well, for categorizing audio files as speech, music and other [15], for speaker verification [16, 17], for emotion recognition [18], for determining food type from eating sounds [19], and for identifying Parkinson's disease [20] and depression [21].

We train our Support Vector Regression (SVR) models on the original, longer segments, and evaluate them on the short chunks. In the absence of chunk-level manual annotation, we compare the utterance-level ground truth scores with the corresponding predictions as well as with their mean. Our main finding is that, while the original chunk-level predictions are actually pretty much uncorrelated with the utterance-level manual annotation scores, using the means of these predictions instead lead to quite high correlation values. We explain this contradiction by the heterogeneous nature of the original (longer) recordings. Indeed, the original utterances tend to consist of parts with remarkably different conflict levels, which are combined into one score by the human annotators; and according to our results, the SVR models could distinguish between these regions when making predictions from the small chunks alone.

In the last part of our study, we also verify this hypothesis by comparing the automatic predictions with human-annotated conflict intensity opinion scores of small chunks. That is, one subject annotated 5-second-long chunks of 100 utterances as well as the full 30-second-long original clips. We measured very similar correlation coefficient, MSE and RMSE values for both utterance length cases. This also supports that the SVR model, trained on 30-second-long utterances, was able to precisely estimate the conflict intensity of much shorter and diverse speech segments. Since in a real-life scenario, automatic conflict detection systems are expected to react within a relatively short amount of time (i.e. a few seconds), this finding might assist actual applications, and bring closer automatic human conflict detection systems to human expectations. 


\section{Fisher Vector Representation}

One of the more frequent problems encountered in computational paralinguistics is that one has to be able to extract a fixedsize feature vector from the utterances, regardless of their duration. Although the utterances can be readily split into very short, overlapping segments (i.e. the frames), utterances of different lengths will also contain a different number of frame-level feature vectors. A good solution for resolving this contradiction and extracting the same number of features from each utterance is to apply Fisher vectors [19, 22].

The aim of the Fisher vector representation is to combine the generative and discriminative machine learning approaches by deriving a kernel from a generative model of the data [14]. That is, let $X=\left\{x_{1}, \ldots, x_{T}\right\}$ be $d$-dimensional low-level feature vectors extracted from an input sample, and let their distribution be modelled by a probability density function $p(X \mid \Theta)$, $\Theta$ being the parameter vector of the model. The Fisher score describes $X$ via the gradient $G_{\Theta}^{X}$ of the $\log$-likelihood function; that is,

$$
G_{\Theta}^{X}=\frac{1}{T} \nabla_{\Theta} \log p(X \mid \Theta) .
$$

This gradient function practically corresponds to the direction in which the model parameters (i.e. $\Theta$ ) should be changed to best fit the data. Notice that, at this point, the size of $G_{\Theta}^{X}$ is already independent of the number of low-level feature vectors (i.e. of $T$ ), and it depends only on the number of model parameters (i.e. $\Theta$ ). The Fisher kernel between the sequences $X$ and $Y$ is then defined as

$$
K(X, Y)=G_{\Theta}^{X} F_{\Theta}^{-1} G_{\Theta}^{Y},
$$

where $F_{\Theta}$ is the Fisher information matrix of $p(X \mid \Theta)$, defined as

$$
F_{\Theta}=E_{X}\left[\nabla_{\Theta} \log p(X \mid \Theta) \nabla_{\Theta} \log p(X \mid \Theta)^{T}\right] .
$$

Expressing $F_{\Theta}^{-1}$ as $F_{\Theta}^{-1}=L_{\Theta}^{T} L_{\Theta}$, we get the Fisher vectors as

$$
\mathcal{G}_{\Theta}^{X}=L_{\Theta} G_{\Theta}^{X}=L_{\Theta} \nabla_{\Theta} \log p(X \mid \Theta) .
$$

When we utilize Gaussian Mixture Models to model the distribution of the low-level features (i.e. $p(X \mid \Theta)$ ) and assume a diagonal covariance matrix, the GMM model has $2 \cdot N \cdot(d+1)$ parameters (i.e. means and variances for each Gaussian component in each feature dimension and the priors of the components) overall; by keeping the prior values fixed, the Fisher vector representation of an instance has a length of twice the number of Gaussian components for each feature dimension.

To apply Fisher vectors to audio processing, it is straightforward to use some standard frame-level features (e.g. MFCCs [23]) of the utterances as the low-level features (i.e. $X)$. When using GMMs, a parameter of the method is the number of Gaussian components $(N)$.

\section{Experimental Setup}

\subsection{The SSPNet Conflict Corpus}

The SSPNet Conflict Corpus [3] contains recordings of Swiss French political debates taken from the TV channel "Canal9". It consists of 1430 recordings, 30 seconds each, making a total of 11 hours and 55 minutes. The ground truth level of conflicts was determined by manual annotation performed by volunteers who did not understand French (French-speaking people were excluded from the list of annotators). Each 30-second-long clip was tagged by 10 annotators; in the end each recording was assigned a score in the range $[-10,10], 10$ denoting a very high
Table 1: Some key properties of the SSPNet Conflict Corpus.

\begin{tabular}{l||c|c|c} 
Set & $\begin{array}{c}\text { No. of } \\
\text { Clips }\end{array}$ & $\begin{array}{c}\text { Total } \\
\text { Duration }\end{array}$ & $\begin{array}{c}\text { Conflict } \\
\text { Scores }\end{array}$ \\
\hline \hline Training & 793 & $6: 36: 13$ & $-0.68 \pm 3.98$ \\
Development & 240 & $2: 00: 00$ & $-0.21 \pm 3.75$ \\
Test & 397 & $3: 18: 16$ & $-0.58 \pm 3.98$ \\
\hline Total & 1430 & $11: 54: 29$ & $-0.58 \pm 3.94$
\end{tabular}

level of conflict and -10 denoting the absence of conflicts. Although the database contains both audio and video recordings, following previous studies (see e.g. [9, 11, 24, 25, 26]), we will rely on the audio data only, and discard the video track.

The audio clips of this dataset were later used in the Conflict sub-challenge of the Interspeech 2013 Computational Paralinguistic Challenge (or ComParE 2013 [27]). Besides completely discarding video data, other steps were made to standardize the work on this dataset, and this setup has since been adopted by most researchers. Perhaps the most important one was that, instead of relying on cross-validation as Kim et al. did when introducing this corpus [3], separate training and test sets were defined. For some key properties of the SSPNet Conflict Corpus, see Table 1 . Note that, although having 12 hours of audio data is nowadays regarded as a quite small dataset in automatic speech recognition, in conflict intensity estimation and in similar areas (commonly known as computational paralinguistics) this counts as a moderate-sized corpus.

\subsection{Technical Parameters}

For the frame-level feature vectors, we used the ComParE feature set proposed by Schuller et al. [27]. It consists of four energy-related features (including loudness, energy and ZeroCrossing-Rate), 55 spectral attributes (e.g. MFCCs, spectral energies and variances, skewness, kurtosis) and 6 voicing-related ones (like $F_{0}$, probability of voicing, logarithmic Harmonicto-Noise Ratio, Jitter and Shimmer). These 65 frame-level attributes and their first-order derivatives were calculated using the OpenSMILE tool [28]. The number of Gaussian Components was $N=2,4,8,16,32,64$ and 128 .

For regression, we employed Support Vector Regression (SVR), using the libSVM implementation [29]. We used a linear kernel, and the $C$ complexity parameter was set in the range $10^{\{-5, \ldots, 1\}}$. Under these experimental conditions, in our preliminary tests we found $N=32$ and $C=10^{-4}$ to be the optimal hyper-parameter values on the development set. Since our study focuses on the applicability of SVR models in very short-term conflict intensity detection, we will employ this SVR model from now on. We would also like to add that this model is an effective one: the correlation coefficient of 0.854 obtained on the test set is a very competitive one, as the highest such score ever reported for the SSPNet Conflict Corpus was 0.856 [12].

Similarly to more recent studies on conflict intensity estimation (e.g. $[10,12,13]$ ), we treat the task as a regression one, and evaluate the performance of a machine learning model primarily via the Pearson's correlation coefficient (CC) of the hand-annotated labels and the predictions. We also report Mean Squared Error (MSE) and Root-Mean Squared Error (RMSE) values. We split the original 30 -second-long utterances of the test set into 1, 2, 3, 5, 10 and 15-seconds-long chunks. 

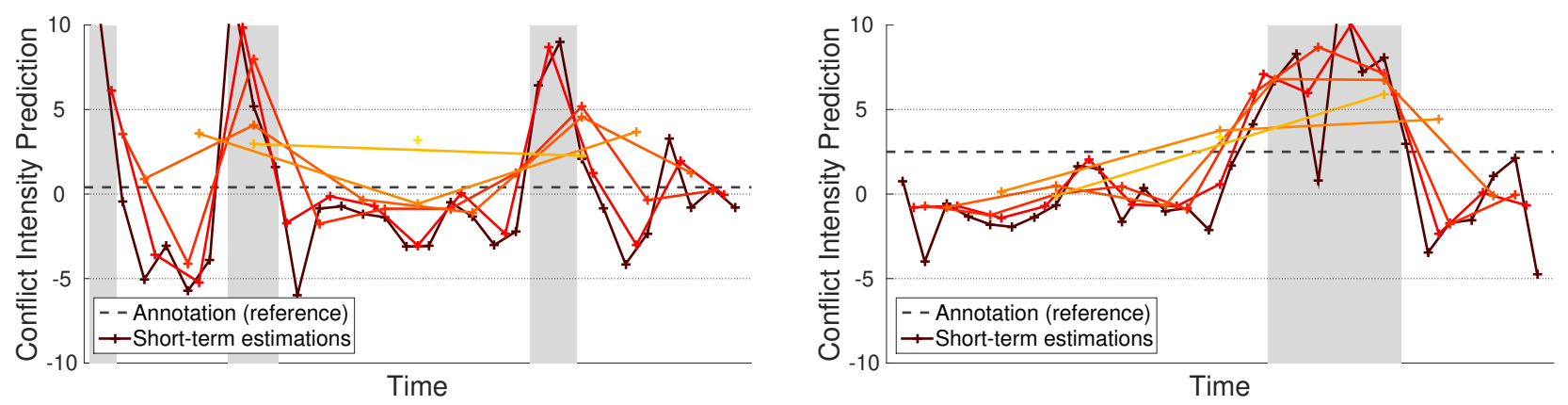

Figure 1: Short-term conflict intensity estimates for two sample utterances. Human-annotated conflict scores are shown with a dashed line, while (also human-annotated) speaker overlap is shown with a gray background.

Table 2: CC, MSE and RMSE values measured on the test set of the SSPNet Conflict corpus, when comparing the predictions with the reference annotation of the full segments.

\begin{tabular}{l||c|c|c}
\multicolumn{1}{c||}{} & \multicolumn{3}{c}{ Prediction Accuracy } \\
\cline { 2 - 4 } Utterance Length & CC & MSE & RMSE \\
\hline \hline 30 sec. (original) & 0.854 & 4.433 & 2.105 \\
\hline 15 sec. & 0.771 & 6.511 & 2.552 \\
10 sec. & 0.719 & 7.685 & 2.772 \\
5 sec. & 0.652 & 9.125 & 3.021 \\
3 sec. & 0.612 & 9.942 & 3.153 \\
2 sec. & 0.580 & 10.556 & 3.249 \\
1 sec. & 0.530 & 11.473 & 3.387
\end{tabular}

\section{Experimental Results}

In our first experiment we made the straightforward assumption that the reference conflict level of each chunk is the same as the reference annotated score of the original, 30-second long utterance (especially since we have no separate chunk-level annotations). Table 2 shows the results obtained in this way. Clearly, the shorter the chunks, the less precise the conflict intensity estimation becomes: while we achieved a CC value of 0.854 for the original segments, we measured a quite low score of 0.530 when we evaluated our regression model on one-second-long chunks. The MSE and RMSE values display similar tendencies: there is a significant increase for the shorter chunks. Considering only these scores, we might have the impression that the low performance associated with the shorter chunks is due to the different conditions (i.e. utterance lengths) of the training and test sets.

A possible different explanation, however, is that the utterances are made up of smaller parts, which have different levels of conflicts, and the human annotators somehow summarize these different conflict intensity values into one score, characterizing the average conflict present in the whole utterance. Therefore, our automatic predictions, obtained only on specific parts of these utterances, cannot reflect the conflict intensity of the whole recording; however, it would make sense to combine these predictions in some way. To do this, we simply took the mean of the SVR outputs corresponding to the chunks of each original utterance. (Note that, to adjust the scale of the predictions, we linearly transformed them so as to have the same mean and standard deviation as the original utterances of the training set; the transformation parameters were set on the dev set.)

The CC, MSE and RMSE scores for the averaged predictions are listed in Table 3. Surprisingly, we see that the
Table 3: CC, MSE and RMSE values measured on the test set of the SSPNet Conflict corpus, when comparing the averaged predictions with the reference annotation of the full segments.

\begin{tabular}{l||c|c|c}
\multicolumn{1}{c||}{} & \multicolumn{3}{c}{ Prediction Accuracy } \\
\cline { 2 - 4 } \multicolumn{1}{c}{ Utterance Length } & CC & MSE & RMSE \\
\hline \hline 30 sec. (original) & 0.854 & 4.433 & 2.105 \\
\hline 15 sec. & 0.850 & 5.635 & 2.374 \\
10 sec. & 0.847 & 5.395 & 2.323 \\
5 sec. & 0.844 & 5.042 & 2.245 \\
3 sec. & 0.843 & 4.875 & 2.208 \\
2 sec. & 0.844 & 4.805 & 2.192 \\
1 sec. & 0.845 & 4.779 & 2.186
\end{tabular}

mean of the predictions for the small audio chunks actually fell quite close to the predictions obtained on the whole utterances. The Pearson's correlation coefficient values fell in the range $[0.843,0.850]$, which means only a slight decrease in performance from the original 0.854 score, while the MSE values rose from 4.433 to $4.779 \ldots 5.635$ and the RMSE values from 2.105 to $2.186 \ldots 2.374$, which are also quite close to the level of the original predictions. In our opinion, this finding supports our observation that the difference between the predictions obtained for the chunks of the same utterances comes from the different actual conflict level of the chunks. Therefore the SVR outputs might actually reflect the conflict level of each chunk more precisely than the manual annotation (which was obtained for the whole 30-second-long utterances).

Fig. 1 shows two sample utterances, and the shortterm predictions obtained for the chunks that have different lengths. Clearly, the predictions show that the SVR models treated the different chunks as ones with different conflict levels: in the first utterance (06-04-12_2400_2430), there are three short intervals that contain a more intense debate than the remaining parts, while for the second utterance (08-02-06_1530_1560) the second half was identified as a higher conflict part than the first half. Interestingly, the (humanannotated) speaker overlaps, shown as grey bars, also indicate that these regions are more intense conflict-wise.

The predictions obtained for the different chunk lengths behave quite similarly, which, in our opinion, also indicates the robustness of the short-term predictions. When using chunks that are only one second long, the curve becomes somewhat jagged, but even this noise leads only to a predicted score difference of 1 or 2 in most cases. 

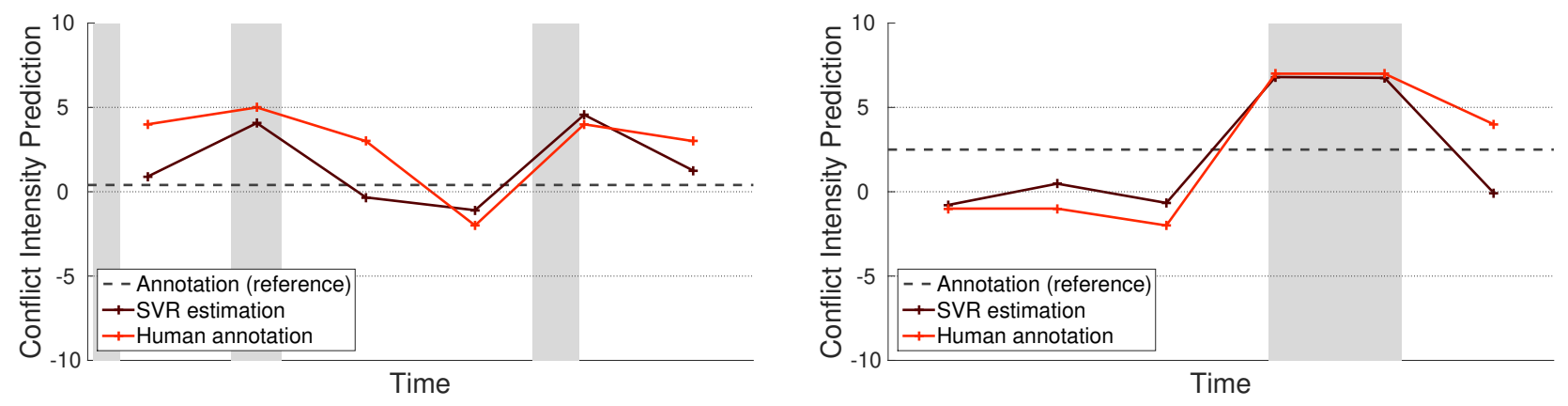

Figure 2: Short-term conflict intensity estimates and manually annotated scores of one annotator for the 5-second-long chunks of the same two utterances as in Fig. 1.

\section{Evaluating versus a Manual Annotation}

Up to now, we argued that Support Vector Regression machine learning models trained on the Fisher vector features are suitable for very short-term conflict intensity estimation, as they were able to find those parts within longer utterances that had different levels of conflicts. In the last part of our study, we investigate this result by comparing the SVR outputs with manually annotated scores. Of course, annotating a dataset of this size is extremely labour-intensive; therefore, our annotation was limited in terms of the amount of audio data, the length of the chunks and the number of annotators. Still, we regard it sufficient to verify our finding from another aspect.

The annotation process covered the first 100 utterances of the test set of the SSPNet Conflict corpus; since the order of the recordings is shuffled in the official file lists, these recordings already showed a great variety of recording dates, topics and speakers. We had only one annotator, the sole author of this study (who, in accordance with the original annotation guidelines, did not understand French ${ }^{1}$ ). Besides ranking the whole 30 second-long utterances in the $[-10,10]$ scale, we split these utterances into chunks with a length of 5 seconds. During annotation, we made sure that all chunks of the same recording were rated in one session, but not directly after each other.

Table 4 shows the CC, MSE and RMSE scores obtained from this experiment. Of course, since we only used 100 utterances in these tests, even the SVR predictions' scores differ slightly compared to those listed in Table 3 . Surprisingly, we found that, either when we compared the scores of the original, 30 second-long utterances or the mean values of the 5 secondlong chunks to the reference scores of the dataset, the SVR predictions always proved to be more similar to these gold standard scores than the opinions of our single human annotator. Of course, due to the inevitable subjectivity present in the annotation process, this does not mean that the SVR model could actually exceed human performance. (Recall that the reference values were determined as the mean ratings of ten annotators for each recording.)

Comparing the conflict intensity values obtained by SVR and by our actual subject (see the last two rows of Table 4), we measured similar CC, MSE and RMSE values in the 5-second and the 30-second cases. Since these manually set scores were obtained in an identical way, we think that this experiment supports our finding that the Support Vector Regression models were able to estimate the intensity of conflicts from a quite short amount of audio data. Fig. 2, which shows the human-annotated

${ }^{1}$ honestly, not a word
Table 4: CC, MSE and RMSE values measured on the first 100 utterances of the test set for the SVR predictions and the humanannotated values compared to the reference annotation (first 4 rows), and their relation (last 2 rows)

\begin{tabular}{l|l||r|r|c}
\multirow{2}{*}{$\begin{array}{c}\text { Utterance } \\
\text { Length }\end{array}$} & \multirow{2}{*}{\multicolumn{1}{c||}{ Approach }} & \multicolumn{3}{c}{ Prediction Accuracy } \\
\cline { 3 - 5 } & SVR & CC & MSE & RMSE \\
\hline \hline \multirow{2}{*}{$30 \mathrm{~s}$} & Human annot. & 0.737 & 4.981 & 2.232 \\
& SVR & 0.830 & 12.114 & 3.481 \\
\hline \multirow{2}{*}{$5 \mathrm{~s}$ (mean) } & Human annot. & 0.789 & 6.545 & 2.430 \\
& SVR vs. Human & 0.783 & 11.393 & 3.358 \\
\hline \hline \multirow{2}{*}{$30 \mathrm{~s}$} & SVR vs. Human & 0.754 & 10.667 & 3.266
\end{tabular}

and automatically predicted scores for the same two utterances as Fig. 1 does, also shows that the two approaches (i.e. SVR and our human annotator) led to remarkably similar estimations.

\section{Conclusions}

In this study we focused on continuous-scale conflict intensity estimation from audio. Although previous works operated only on longer segments, now we have shown that Support Vector Regression models, using Fisher vectors as features, are capable of determining conflict level from much shorter audio chunks. Although the predictions for these small chunks of the SVR model were only loosely correlated with the reference conflict scores obtained via human annotation, it turned out that it was due to the fact that these subsegments of the original, much longer utterances indeed contained conversational parts with different conflict levels. We also verified this finding by annotating 5-second-long chunks created from a subset of the publicly available SSPNet Conflict corpus. Our findings might help actual conflict detection applications by allowing them to process very short audio data, hence reduce their reaction time and so bring closer their operation to human expectations.

\section{Acknowledgements}

This research was partially supported by the Ministry for Innovation and Technology, Hungary (grants TUDFO/471381/2019-ITM and the New National Excellence Program ÚNKP20-5), and by the National Research, Development and Innovation Office of Hungary via contract NKFIH FK-124413. G. Gosztolya was also funded by the János Bolyai Scholarship of the Hungarian Academy of Sciences. 


\section{References}

[1] J. L. Hocker and W. W. Wilmot, Interpersonal Conflict. Brown \& Benchmark, 1995.

[2] J. Z. Rubin, D. G. Pruitt, and S. H. Kim, Social conflict: Escalation, stalemate, and settlement. McGraw-Hill Book Company, 1994.

[3] S. Kim, F. Valente, M. Filippone, and A. Vinciarelli, "Predicting continuous conflict perception with Bayesian Gaussian Processes," IEEE Transactions on Affective Computing, vol. 5, no. 2, pp. 187-200, 2014.

[4] P. Spector and S. Jex, "Development of four self-report measures of job stressors and strain: interpersonal conflict at work scale, organizational constraints scale, quantitative workload inventory, and physical symptoms inventory," Journal of Occupational Health Psychology, vol. 3, no. 4, pp. 356-367, 1998.

[5] N. Ferguson, "Simultaneous speech, interruptions and dominance," British Journal of Social and Clinical Psychology, vol. 16, pp. 295-302, 1977.

[6] V. W. Cooper, "Participant and observer attribution of affect in interpersonal conflict: an examination of noncontent verbal behavior," Journal of Nonverbal Behavior, vol. 10, pp. 134-144, 1986.

[7] S. Kim, F. Valente, and A. Vinciarelli, "Automatic detection of conflicts in spoken conversations: Ratings and analysis of broadcast political debates," in Proceedings of ICASSP, Kyoto, Japan, 2012, pp. 5089-5092.

[8] Y. Panagakis, S. Zafeiriou, and M. Pantic, "Audiovisual conflict detection in political debates," in Proceedings of ECCV, Zurich, Switzerland, 2014, pp. 304-314.

[9] M.-J. Caraty and C. Montacié, Detecting Speech Interruptions for Automatic Conflict Detection. Springer International Publishing, 2015, ch. 18 , pp. 377-401

[10] G. Gosztolya, "Conflict intensity estimation from speech using greedy forward-backward feature selection," in Proceedings of Interspeech, Dresden, Germany, Sep 2015, pp. 1339-1343.

[11] C. Georgakis, Y. Panagakis, S. Zafeiriou, and M. Pantic, "The Conflict Escalation Resolution (CONFER) database,' Image and Vision Computing, vol. 65, no. 1, pp. 37-48, 2017.

[12] G. Gosztolya and L. Tóth, "DNN-based feature extraction for conflict intensity estimation from speech," IEEE Signal Processing Letters, vol. 24, no. 12, pp. 1837-1841, 2017.

[13] V. Rajan, A. Brutti, and A. Cavallaro, "ConflictNET: End-to-end learning for speech-based conflict intensity estimation," IEEE Signal Processing Letters, vol. 26, pp. 1668-1672, 2019.

[14] T. S. Jaakkola and D. Haussler, "Exploiting generative models in discriminative classifiers," in Proceedings of NIPS, Denver, CO, USA, 1998, pp. 487-493.

[15] P. J. Moreno and R. Rifkin, "Using the Fisher kernel method for web audio classification," in Proceedings of ICASSP, Dallas, TX, USA, 2010, pp. 2417-2420.

[16] Y. Tian, L. He, Z. yi Li, W. lan Wu, W.-Q. Zhang, and J. Liu, "Speaker verification using Fisher vector," in Proceedings of ISCSLP, Singapore, Singapore, 2014, pp. 419-422.

[17] Z. Zajíc and M. Hrúz, "Fisher vectors in PLDA speaker verification system," in Proceedings of ICSP, Chengdu, China, 2016, pp. 1338-1341.

[18] G. Gosztolya, "Using the fisher vector representation for audio-based emotion recognition," Acta Polytechnica Hungarica, vol. 17 , no. 6 , pp. 7-23, 2020.

[19] H. Kaya, A. A. Karpov, and A. A. Salah, "Fisher Vectors with cascaded normalization for paralinguistic analysis," in Proceedings of Interspeech, 2015, pp. 909-913.

[20] J. V. Egas López, J. R. Orozco-Arroyave, and G. Gosztolya, "Assessing Parkinson's disease from speech by using Fisher Vectors," in Proceedings of Interspeech, Graz, Austria, Sep 2019, pp. 3063 3067.
[21] V. Jain, J. L. Crowley, A. Dey, and A. Lux, "Depression estimation using audiovisual features and Fisher Vector encoding," in Proceedings of ACM Multimedia, Nov 2014, pp. 87-91.

[22] G. Gosztolya, "Using Fisher Vector and Bag-of-Audio-Words representations to identify Styrian dialects, sleepiness, baby \& orca sounds," in Proceedings of Interspeech, Graz, Austria, Sep 2019, pp. 2413-2417.

[23] L. Rabiner and B.-H. Juang, Fundamentals of Speech Recognition. Prentice Hall, 1993.

[24] O. Räsänen and J. Pohjalainen, "Random subset feature selection in automatic recognition of developmental disorders, affective states, and level of conflict from speech," in Proceedings of Interspeech, Lyon, France, Sep 2013, pp. 210-214.

[25] R. Brueckner and B. Schuller, Be at Odds? Deep and Hierarchical Neural Networks for Classification and Regression of Conflict in Speech. Springer International Publishing, 2015, ch. 19, pp. 403 429.

[26] H. Kaya, T. Özkaptan, A. A. Salah, and F. Gürgen, "Random discriminative projection based feature selection with application to conflict recognition," IEEE Signal Processing Letters, vol. 22, no. 6, pp. 671-675, 2015.

[27] B. W. Schuller, S. Steidl, A. Batliner, A. Vinciarelli, K. Scherer, F. Ringeval, M. Chetouani, F. Weninger, F. Eyben, E. Marchi, H. Salamin, A. Polychroniou, F. Valente, and S. Kim, "The Interspeech 2013 Computational Paralinguistics Challenge: Social signals, Conflict, Emotion, Autism," in Proceedings of Interspeech, Lyon, France, Sep 2013, pp. 148-152.

[28] F. Eyben, M. Wöllmer, and B. Schuller, "Opensmile: The Munich versatile and fast open-source audio feature extractor," in Proceedings of ACM Multimedia, 2010, pp. 1459-1462.

[29] C.-C. Chang and C.-J. Lin, "LIBSVM: A library for support vector machines," ACM Transactions on Intelligent Systems and Technology, vol. 2, pp. 1-27, 2011 